\section{What can we do to prepare patients for test results during pregnancy?}

Screening for Down syndrome, neural tube defects, and trisomy 18 with maternal serum AFP is considered by some to be a routine part of pregnancy care. The American College of Obstetrics and Gynecology and the United States Preventive Services Task Force recommend offering maternal serum AFP screening to all pregnant women between 16 and 18 weeks of their pregnancy. ${ }^{1}$

The interval between having a positive screening test result and finding a definitive answer is stressful. According to Rausch and colleagues, this anxiety was the cause of decreased participation in maternal serum screening in subsequent pregnancies. The study suggests that providers of maternity care do not do enough to prepare women for the possibility of a false-positive test result. Anxiety about abnormal test results is the opposite of what women desire from prenatal testing. Expectant women often tell me that they just want to know that their baby is normal. Patients are anxious after having a positive test result, and the anxiety often remains even after they learn that the result was falsely positive.
John E Delzell, Jr

Department of Family and Community Medicine MA303 Health Sciences

Center

University of Missouri-Columbia Columbia, MO 65203

Correspondence to: Dr Delzell Delzellj@health.missouri. edu

Competing interests: None declared

West J. Med 2000;173:183-184 
It is interesting that maternal serum AFP testing has become a de facto standard of care without the evidence that would typically be required to support a screening test. A recent article by Rogers reminds us that a screening test should follow the principle of primum non nocere: "first do no harm." 2 Physicians should consider 3 elements before offering a screening test. ${ }^{3}$

The burden of suffering from a disease is the prevalence of the disease in combination with the morbidity or mortality associated with that disease. The burden associated with the genetic diseases that maternal serum AFP screening can detect is certainly high enough to justify screening. Neural tube defects, for example, occur in 3 to 4 per 10,000 live births - a relatively common genetic disorder.

The screening test must be effective. The test should minimize the number of false-positive test results while maximizing the true-positive results. The likelihood that a positive test result means that someone has the disease (known as the positive predictive value) should be high. The test should not be prohibitively expensive, and the side effects of the screening test should be minimal. One of the problems with maternal serum AFP testing is the high number of false-positive results. Most women who test positive will have a normal fetus. The consequences of a positive test result may include amniocentesis, ultrasonography, and increased fetal surveillance. Other harm from a positive screening test result can include labeling, anxiety, and the increased costs associated with the additional workup.

The third element of a screening test is the evidence that screening actually prevents morbidity or mortality.
With prenatal screening, this is critical because finding some disorders earlier in pregnancy-for example, intrauterine growth retardation-can affect the management of the pregnancy. No trials have shown improved outcomes of patients who have undergone screening for Down syndrome or neural tube defects.

So, what should physicians do with obstetric patients? Rausch and associates have documented the effects of false-positive screening test results during pregnancy on patient anxiety. It is important to obtain informed consent before ordering maternal serum AFP screening. Patients should understand the risks of false-positive and -negative test results and should know the consequences of a positive result and subsequent testing options. After they have received all of the information, they can make an informed choice about whether or not to have the test. Physicians should also counsel patients adequately about their results. We have a responsibility to evaluate the evidence behind screening tests before we offer them and to prepare obstetric patients for the "harm" that they may cause.

Dr Delzell cochairs the Group on Family-Centered Perinatal Care for the Society of Teachers of Family Medicine.

References

1 US Preventive Services Task Force. Guide to Clinical Preventive Services. 2nd ed. Baltimore: Williams \& Wilkins; 1996.

2 Rogers J. Clinical preventive medicine: primum non nocere? J Fam Pract 2000;49:442-444.

3 Barratt A, Irwig L, Glasziou P, et al. Users' guides to the medical literature, XVII: how to use guidelines and recommendations about screening. Evidence-Based Medicine Working Group. JAMA 1999;281:2029-2034.

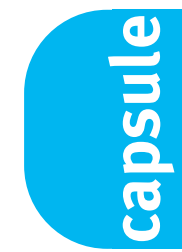

Walnuts and macadamia nuts benefit lipid profiles Like walnuts, macadamia nuts can improve serum lipid profiles (Arch Intern Med 2000;160:1154-1158). Although a macadamia nut is $75 \%$ fat, most of the fat is monounsaturated. In a crossover experiment, replacing the $37 \%$ fat in a typical US diet with fat from macadamia nuts had a statistically significant impact on serum lipid parameters.

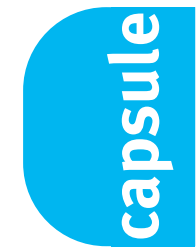

Acupressure bands don't prevent nausea It would be wonderful if a simple acupressure wristband reduced nausea and vomiting after surgery, but it doesn't seem to (Can J Anaesth 2000;47:319-324). A large randomized trial comparing the real thing with a sham wristband after endoscopic urological procedures found no difference between groups. Other investigators have had more luck with acupuncture, but it's more invasive and therefore less convenient. 